\section{Acrylamid-Exposition: höhere Mortalität}

Acrylamid ist ein bekanntes Kanzerogen. In einer prospektiven Kohortenstudie wurde erstmals untersucht, ob eine prädiagnostische Acrylamidexposition die Mortalität von postmenopausalem Brustkrebs beeinflusst.

/ on 1993 bis 1997 wurden 79.729 dänische Frauen im Alter zwischen 50 und 64 Jahren ohne MammakarzinomDiagnose zur Teilnahme an der prospektiven Studie eingeladen. Sie sollten u.a. Fragen zum Lebensstil und zur Hormonsubstitution beantworten, von jeder Frau wurden Blutproben eingefroren. Zur Auswertung kamen die Daten von 24.697 Frauen. 420 von ihnen entwickelten vor 2001 einen Brustkrebs und 110 starben vor 2009.

Im Vergleich zur Gesamtzahl aller in der Kohorte aufgetretenen Brustkrebsfälle hatten die an der Krankheit verstorbenen Frauen weniger häufig eine Hormonsubstitution erhalten und weni- ger geraucht. Die in der Blutprobe nachgewiesenen Addukte von Hämoglobin mit Acrylamid bzw. mit seinem wichtigsten Metaboliten Glycidamid (AA-Hb und GA-Hb) korrelierten in der Gesamtgruppe nicht eindeutig mit der Mortalität.

Bei den Nichtraucherinnen waren höhere GA-Hb-Konzentrationen mit einem höheren Risiko für krebsspezifischer Mortalität assoziiert (Hazard Ratio [HR] 1,63, 95\%-Konfidenzintervall [95\%-KI] 1,06-2,51), die HR bei den Frauen mit einem Östrogenrezeptor-positiven Tumor betrug 2,23 (95\%-KI 1,38-3,61).

Beim AA-Hb-Addukt ergab sich ein ähnliches Bild, statistische Signifikanz wurde hier aber nur bei Frauen mit einem Rezeptor-positiven Brustkrebs gefunden (HR 1,31, 95\%-KI 1,02-1,69). Bei den Frauen, die während der Phase der Blutabnahme geraucht hatten, waren keine eindeutigen Zusammenhänge zwischen Mortalität und Acrylamidexposition zu erkennen. Allerdings schien auch bei diesen Frauen eine höhere GA-HbKonzentration zusammen mit einem positiven Hormonrezeptorstatus die Mammakarzinom-Mortalität zu erhöhen.

Fazit: Eine prädiagnostische AcrylamidExposition kann wahrscheinlich vor allem bei Frauen mit einem hormonrezeptorpositiven Mammakarzinom die krankheitsspezifische Mortalität erhöhen.

Barbara Kreutzkamp

Olsen A et al. Pre-diagnostic acrylamid exposure and survival after breast cancer among postmenopausal Danish women. Toxicology. 2012; 296(1-3):67-72.

\section{Ist moderne Bildgebung die häufigste vermeidbare Ursache für Brustkrebs?}

Nach einer Untersuchung des US-amerikanischen Institute of Medicine (IOM) gibt es zwei Umweltfaktoren, die die Entstehung von Brustkrebs besonders fördern: kombinierte Hormonersatztherapie und die Exposition gegenüber ionisierender Strahlung. Ein hohes Präventionspotenzial sehen die IOMExperten vor allem in der Beschränkung von CT-Untersuchungen.

D as unabhängig arbeitende IOM hat den Einfluss von Umweltfaktoren auf das Brustkrebsrisiko nach den Maßgaben der evidenzbasierten Medizin untersucht. Für eine Vielzahl der üblichen Verdächtigen konnte interessanterweise kein eindeutiger Zusammenhang mit dem Brustkrebsrisiko nachgewiesen werden: In Konsumgütern enthaltene Chemikalien wie Bisphenol A und Phthalate, Industriechemikalien wie Benzol und Ethylenoxid sowie Pestizide wie DDT. Allerdings räumt das IOM ein, dass für viele dieser Substanzen die Datenlage noch dünn ist.

Mit ausreichender Evidenz untermauert ist laut IOM, dass die kombinierte Hormonersatztherape und ionisierende Strahlung das Brustkrebsrisiko stärker beeinflussen als andere Umweltfaktoren. Den Nachweis für die brustkrebsför- dernde Wirkung einer postmenopausalen Östrogen-Gestagen-Therapie hat vor zehn Jahren die Women's Health Initiative erbracht. Seitdem ist der Gebrauch der Hormonersatztherapie stark zurückgegangen - und die Brustkrebsinzidenz in der Folge leicht gesunken.

Gemäß dem IOM-Bericht ist daher das Vermeiden von unnötiger Strahlenbelastung durch computertomografische Untersuchungen einer der wichtigsten Schritte, die Frauen unternehmen können, um ihr Brustkrebsrisiko zu senken. Die Strahlenbelastung bei einer Thorax-CT ist 100- bis 500-mal so hoch wie bei einer konventionellen RöntgenThorax-Untersuchung. Das IOM schätzt, dass in den USA pro Jahr 2.800 Brustkrebsfälle auf das Konto ionisierender Strahlung gehen, davon etwa 1.900 auf das von Computertomografien. Dies sei

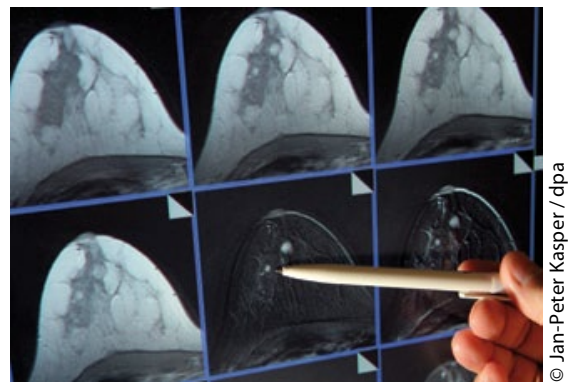

CT-Untersuchungen im Thoraxgebiet: nach Möglichkeit vermeiden.

zwar nur ein kleiner Anteil der Brustkrebsfälle, aber insofern bedeutend, weil viele von ihnen vermeidbar seien. Selbst nach Aussagen führender Radiologen seien mehr als $30 \%$ der bildgebenden Untersuchungen nicht notwendig.

Darüber hinaus kann das Brustkrebsrisiko laut IOM-Bericht in bescheidenem Maße auch durch den Lebensstil modifiziert werden. Frauen, die nur wenig Alkohol trinken, ein gesundes Körpergewicht halten und nicht rauchen, erkranken seltener an Brustkrebs.

Beate Schumacher

Smith-Bindman R. Environmental causes of breast cancer and radiation from medical imaging. Findings from the Institute of Medicine report. Arch Intern Med. 2012;172(13):1023-7. 